\title{
Is There Improvement in the Care for Patients With Multiple Myeloma?
}

\author{
Svetlana Stankovikj ${ }^{1}$, Kata Martinova ${ }^{2}$, Vera Stankovikj ${ }^{3}$
}

\begin{abstract}
Improved understanding of the multiple myeloma biology along with the discovery of novel anti-myeloma agents has led to a better-quality treatment of these patients. Multiple myeloma can cause a variety of complications such as hyperviscosity syndrome, hypercalcaemia, spinal cord compression, early infection, bone disease and renal impairment, some of which are life-treatening. Treatment of these has been demonstrated to significantly reduce morbidity and early fatal outcome. Utilization of novel agents including: Thalidomide, Bortezomib and Lenalidomide as part of the induction protocols before autologous stem cell transplantation (ASCT) and also as a front-line treatment in older patients, has been shown to be superior in comparison with the previous treatment strategies. The latter has been confirmed regarding the overall treatment response, progression-free survival and even better overall survival in some studies. Greater experience in dosing of these medications has led to a decrease in the toxicity of the protocols. A third phase clinical study has demonstrated a better clinical outcome when Bortezomib was used prior ASCT. Furthermore, involvement of these agents in the post-transplant consolidation and maintenance treatments has been shown to be beneficial in other aspects such as prolongation of progression-free survival. The immune therapy represents a potentially promising treatment modality. Daratumumab is a new, specific, human monoclonal antibody with high affinity to a unique epitope on CD38. It induces plasma cells death through several immunological mechanisms. It has a good safety profile and a substantially activity as a single-agent therapy in refractory and relapsed myeloma.
\end{abstract}

Keywords: multiple myeloma, ASCT, Bortezomib, Thalidomide, Daratumumam

\section{Introduction}

Multiple myeloma is a malignant disorder of plasma cells, characterized by uncontrolled and progressive proliferation of a single clone of plasma cells. The disease leads to progressive morbidity and eventual mortality by lowering resistance to infection and causing significant skeletal destruction with bone pain, pathological fractures and hypercalcemia. The other disease characteristics are anemia, renal failure, neurological complications and hyperviscosity syndrome. Most of the patients produce a monoclonal protein so-called paraprotein, M-protein or M-component, which is an immunoglobulin (Ig) or a fragment of one that has lost its function (1). The normal immunoglobulin levels are compromised, leading to predilection to infections. The proliferated plasma cells displace the normal bone marrow architecture, which is reflected by clinical findings such as anemia, neurological symptoms and bone resorption seen as diffuse osteoporosis or lytic bone lesions (2). Furthermore, hypercalcemia, renal impairment and detectable monoclonal protein in the serum and/or urine are frequently seen. A small minority of patients are non-secretory.

At the time of diagnosis, $\mathrm{MM}$ is a heterogeneous disease, with a course that varies on the basis of both disease and host-related factors (age, renal function, stage, chromosomal abnormalities etc.). Multiple myeloma causes significant morbidity and mortality. It accounts for approximately $1 \%$ of all malignancies and 13\% of hematological cancers. Approximately 50000 patients per year are diagnosed with MM in EU and US, and 30000 patients per year die due to multiple myeloma $(4,5)$.

\section{Treatment Approach}

MM can cause many acute complications such as hyperviscosity syndrome, hypercalcemia, spinal cord compression, early infection, bone disease and renal impairment; some of them are life-threatening. The treatment of these medical emergencies has greatly decreased morbidity and early mortality in patients.

Hyperviscosity syndrome occurs in cases with high serum paraprotein levels, particularly those of IgA or IgG3 type. Symptoms include: blurred vision, headaches, mucosal bleeding and dyspnea due to heart failure. Symptomatic patients should be treated urgently with plasma exchange; isovolaemicvenesection may be useful if plasma exchange facilities are not immediately available. If transfusion is essential, exchange transfusion should be performed. Rapid reduction of protein levels is mandatory and anti-myeloma treatment should be instituted promptly. (6).

Hypercalcemia occurs in about $30 \%$ of newly diagnosed patients. Acute hypercalcemia can present with central nervous system dysfunction (confusion, coma and obtundation), muscle weakness, pancreatitis, constipation, thirst, polyuria, shortening of the Q-T interval on ECG and even, acute renal failure. Mild hypercalcemia with calcium range from $2.6-2.9 \mathrm{mmol} / \mathrm{l}$, may be corrected with oral and/or intravenous rehydration. Moderate and severe hypercalcemia with calcium range over $2.9 \mathrm{mmol} / 1$, requires intravenous rehydration with normal saline. Adequate urine output should be ensured and use of intravenous loop diuretics such as furosemide should be considered to avoid volume overload and heart failure and promote urinary calcium excretion. All patients with moderate to severe hypercalcemia should receive a bisphosphonate (zolendronic acid is the bisphosphonate of choice). Dental procedures, such as root canal or extraction of teeth, may be associated with infection or destruction of the jaw (osteonecrosis), so they should be performed before bisphosphonate treatment is started.

Spinal cord compression can be seen in $5 \%$ of patients with MM. Clinical features depend on the nature of the cord compression (due to bony/structural lesion or soft tissue 


\section{International Journal of Science and Research (IJSR) \\ ISSN (Online): 2319-7064}

Index Copernicus Value (2013): 6.14 | Impact Factor (2014): 5.611

disease), the spinal level, extend of the disease and the rate of development of cord compression and commonly include sensory loss, paresthesiae, limb weakness, walking difficulty and sphincter disturbance. Spinal cord compression is a medical emergency requiring rapid diagnosis and treatment to prevent irreversible damage, such as paralysis. Upon clinical suspicion for such a complication, Dexamethasone 40mg daily for 4 days should be commenced. MRI or at least CT scan should be immediately obtained. If there is a structural compression of spinal cord, it is necessary to perform urgent surgical decompression, usually followed by local radiotherapy (at the latest for 24 hours) with a dose of at least $30 \mathrm{~Gy}$ (8). For soft tissue disease local radiotherapy is the treatment of choice and should be commenced urgently, preferably within 24 hours.

The early infection in patients with MM is related to deficits in both humoral and cellular immunity. It has been reported that up to $10 \%$ of patients die of infective causes early, within 60 days of diagnosis. It is recommended to treat every febrile patient with wide spectrum antibiotics. In case of severe, systemic infections or neuthropenic sepsis, parenteral antibiotics should be administrated but aminoglycosides should be avoided (9).

Bone disease occurs in about $80-90 \%$ of patients with MM. It results in pain, pathological fractures with potential risk of cord compression and hypercalcemia. Skeletal events compromise mobility and independence of patients, decreasing quality of life. Long bone fractures are treated by immobilization and radiotherapy that can improve pain and promote healing of the fracture site. The recommended dose is $8 \mathrm{~Gy}$ for a single fracture. In case of larger lytic lesions of skeleton, an orthopedic surgeon should be consulted and in some patients a surgery with vertebroplasty or kyphoplasty should be done. Bisphosphonates have a favorable effect in controlling the bone lesions in patients with MM, even in those without bone disease at presentation. The duration of the treatment depends on individual factors such as: achieving remission, expressiveness of bone lesions, renal function (10). It is reasonable to stop the treatment in patients who achieve complete response or a very good partial remission and who do not have active bone disease. It is mandatory to perform a good dental inspection always when bisphosphonate therapy is planned and to perform dental procedures such as root canal or extraction of teeth before these agents are started in order to prevent the most difficult complication-osteonecrosis of the jaw.

Renal failure is a frequent and potentially serious complication that occurs in $20-25 \%$ of patients at presentation and in nearly $50 \%$ of patients during the disease course. It is due to destruction of renal tubules from the serum free light chain ("myeloma kidney") but other factors such as dehidratation, hypercalcemia, application of nephrotoxic agents or infections, also, contribute. Patients, who have renal failure at presentation, have high level of early fatal outcomes. For maintenance of a good renal function, patients are advised to stay well-hydrated, to drink enough fluids to produce three liters of urine daily and to avoid nephrotoxic agents including aminoglycosides and non-steroidal anti-inflammatory drugs (11).

\section{Chemotherapeutical Protocols}

Multiple myeloma can remain stable for prolonged periods of time. Individuals with early myeloma who have no symptoms (often called "smoldering" myeloma) may be advised to wait months to years before considering chemotherapy. Regular controls should be performed on 3 months intervals with quantifying serum and urinary Mprotein. Bone marrow examination and bone x-ray should be performed before initiating the chemotherapy. The chemotherapy is indicated for symptomatic myeloma in patients with ROTI (Myeloma related Organ or Tissue Impairment) or CRAB (Calcium levels increased, Renal failure, Anemia, Bone lesions).

The choice of treatment protocol depends on the age of the patient, performance status, aggressiveness of the disease and other prognostic factors (3). Newly diagnosed patients with $\mathrm{MM}$ are categorized in 2 subpopulations, usually depending on their age and the convenience for the following treatment. The younger patients should be treated with induction protocols that will be followed with consolidation with high-dose chemotherapy (HDC) and autologous stem cell transplantation (ASCT). Patients, who are not eligible for HDC and ASCT, should be treated with long-lasting therapy that includes combination of several medications (alkylating agents, high-dose corticosteroids and the new agents).

\section{A. Induction therapy in patients not eligible for HDC and ASCT}

The aim of this treatment is to achieve the maximum, durable response with minimal treatment-related toxicity. Oral MP protocol (Melphalan + Prednisolon) was utilized as a first line treatment from 1960-ies and for a long time remained a gold standard for treating patients not eligible for ASCT. Cyclophosphamide versus Melphalan asserted nearly identical results in $2 \mathrm{UK}$ MRC trials. However, Melphalan was preferred because of its better tolerability in older population. More complex protocols including combination of several intravenous drugs were utilized afterwards. Nevertheless, a large meta-analysis of Myeloma Triallists Collaborative Group from 1998, did not assert higher efficiency related to oral MP protocol $(12,13)$.

Thalidomide is one of the first newly introduced agents for treatment of MM. It is one of the "immunomodulatory" agents that have several mechanisms of action: antiangiogenic effect, inhibition of tumor necrosis factor (TNF), secretion of interleukin 2 and induction of apoptosis of tumor cells. Thalidomide is an efficient agent in all phases of MM treatment. It can be used as a single agent therapy or in combination with steroids or other drugs. The study of Rajkumar et al., 2006, phase III (14) compared TD (Thalidomide + Dexamethasone) protocol to Dexamethasone as a single-agent therapy in patients at middle age of 65 years. It demonstrated significant benefit in relation to response to therapy (overall response $\mathrm{CR}+\mathrm{PR}$ ) in $63 \%$ versus $46 \%$. There was a benefit regarding the time to progression (TP) in TD group but also, grade 3 nonhematological toxicity in $67 \%$ of patients on TD protocol. 


\section{International Journal of Science and Research (IJSR) \\ ISSN (Online): 2319-7064}

Index Copernicus Value (2013): 6.14 | Impact Factor (2014): 5.611

The trial of Ludwig et al. 2009, directly compared TD versus MP protocol. Superior response to treatment was demonstrated in 298 older patients in TD group (68\% versus $50 \%$ ) but similar progression free survival (PFS) and time to progression (TTP) in both groups. Nevertheless, the overall survival was significantly lower in TD group that was probably due to the fact that this much older population of patients $(60 \%$ between 70 and 79 years of age), were not able to tolerate the high doses of Thalidomide and Dexamethasone (15).

Five randomized studies in older patients compared MP versus MPT protocol as first line treatment. They all demonstrated superior response in MPT group : higher percentage of PR, VGPR or near-CR as well as PFS and, significantly longer overall survival (OS) was shown just in two studies (13). The English investigators created alternative to MPT protocol with CTD (cyclophosphamide, thalidomide, dexamethasone), that was the non-invasive arm of Myeloma IX trial. The early evaluation demonstrated superior response in CTD versus MP and similar response with MPT.

Velcade (Bortezomib) belongs to the group of "proteasome inhibitors". As a targeted therapy it acts through blockade and weakening of the function of proteasomes inside the cells. Velcade is used as a single-agent therapy or in combination with Melphalan and Prednisolone (VMP protocol). VMP protocol is usually administrated over one year. VISTA study evaluated the effect of treatment in VMP versus MP protocol in newly diagnosed MM patients that were not eligible for APSCT. The results confirmed the superiority of VMP versus MP regarding the response to treatment $(\mathrm{CR}$ in $30 \%$ versus $4 \%$ and $\mathrm{PR}$ in $71 \%$ versus $35 \%$ ). The study of Palumbo, 2010 compared the effects of VMPT vs. VMP. The induction treatment with VMPT followed by continuous VT treatment, demonstrated superiority in relation to VMP; 3 -yearly PFS 56\% in VMPTVT vs. $41 \%$ in patients receiving VMP protocol (16).

Lenalidomide (Revlimid) belongs to the group of "immunomodulatory drugs" and is analog to Thalidomide, but with different side effects. It reacts as an inhibitor of angiogenesis and in cytokine modulation of T-cell stimulation. Several randomized studies analyzed the effect of Lenalidomide as induction treatment in MM (17). One large randomized study, phase 3 (MM-020/IFM07-01) compared the results of treatment in three arms: arm A (Lenalidomide + low-dose dexamethasone) continually until disease progression, arm B (Lenalidomide + low-dose dexamethasone during 72 weeks, 18 cycles) and arm C (MPT during 72 weeks, 12 cycles). It was shown that the risk for fatal outcome was decreased for $22 \%$ in arm A vs. arm C; ORR and PFS much better in arm A vs. arm C and also better safety profile. In the study of Palumbo et al. 2009, MRP protocol (Melphalan, Prednisolone and Lenalidomide) phase I/II has shown comparable response to treatment with fewer non-hematological side effects.

In Europe, standard treatment for MM patients not eligible for HDC and ASCT remained MPT protocol (MelphalanPrednisolone-Thalidomide) (Fayers 2011) and VMP protocol (Velcade-Melphalan-Prednisolon) (San Miguel 2008).

\section{B. Induction treatment before HDC and ASCT}

Before introducing the new agents, VAD protocol (Vincristine, Adriamycin and Dexamethasone) was the standard treatment for these patients. In the studies of Alexanian et al. 1990, Gore et al.1989 and Sampson et al.1989, response to treatment was shown in $55-84 \%$ of patients with CR in 8-28\% (18). The study of Cavoet al.2005 compared TD protocol (Thalidomide-Dexamethasone) vs. VAD protocol (19). Significantly better response was demonstrated with TD but not superior response in the phase post-ASCT. Myeloma IX trial compared CVAD (Cyclophosphamide, Vincristine, Doxorubicin, Dexamethasone) with CTD (Cyclophosphamide, Thalidomide, Dexamethasone) protocol. Preliminary results showed better response to treatment in CTD arm but there are still no results regarding better PFS and OS (20). CTD is now the most frequently used protocol in Great Britain because it does not affect the mobilization of stem cells. Two large, phase 3 trials, showed that the protocols containing Bortezomib have good results as induction treatment before ASCT. IFM group compared 4 cycles of VAD vs. Bortezomib-Dexamethason in 482 patients at the age of 65 (Harousseau et al.). $\mathrm{CR}$ or $\mathrm{CR} / \mathrm{nCR}$ was significantly better post-ASCT in the arm with Bortezomib, better PFS but not better OS. Several trials with Lenalidomide and Dexamethasone +/- chemotherapy have shown better response. The trial of Rajkumaret al.2005, demonstrated that all patients had passed successful mobilization of sufficient amount of stem cells.

Yet, VAD protocol or Dexamethasone as a single agent therapy, are not recommended as induction treatment before HDC and ASCT, but protocols that contain at least one of the new agents. CTD, TAD (Thalidomide, Doxorubicin, and Dexamethasone), Bortezomib-Dexamethasone and PAD (PS341-Bortezomib-Doxorubicin-Dexamethasone) are recommended. Which protocol to use will depend on several factors such as: renal function, risk of thrombosis and pre-existing neuropathy.

Daratumumab is a humanized IgG1 kappa monoclonal antibody that bands with high affinity to the unique epitope of CD38 trans membrane glycoprotein (21). The treatment with Daratumumab is a targeted immune-therapy directed to tumor cells that have high expression of CD38, such are the plasma cells of the patients with MM. Several clinical trials are ongoing that expect the effect of this treatment: Daratumumab as a single agent therapy or in combination with Lenalidomide-Dexamethasone or with VMP protocol.

\section{Conclusion}

MM remains an incurable disease further more. However, the implementation of the new therapeutically protocols and the ASCT as well as the improvement in symptomatic and supportive care, contributed to enhance response to treatment, to prolong the event free survival and the overall survival and, what is most important, to improve the quality of life of these patients. 


\section{International Journal of Science and Research (IJSR)}

ISSN (Online): 2319-7064

Index Copernicus Value (2013): 6.14 | Impact Factor (2014): 5.611

\section{References}

[1] Kyle RA, Rajkumar SV. Criteria for diagnosis, staging, risk stratification and response assessment of multiple myeloma. Leukemia 2009; 23:3-9.

[2] Kyle RA, Gertz MA, Witzig TE, etal.Review of 1027 patients with newly diagnosed multiple myeloma. MajoClin Proc.2003; 78:21-33.

[3] Palumbo A, Anderson K., Multiple myeloma. N Engl J Med.2011; 364:1046-1060.

[4] American Cancer Society (ACS). Cancer Facts and Figures 2012. Atlanta: American Cancer Society; 2012.

[5] Ferlay J., Shin HR, Bray F., Forman D., Mathers C., Parkin DM. Estimates of worldwide burden of cancer in 2008:GLOBOCAN 2008.Int J Cancer 2010;127:28932917.

[6] Mehta J., Singhah S., (2003) Hyperviscosity syndrome in plasma cell dyscrasias. Seminars in Thrombosis and Hemostasis 29; 467-471.

[7] Rades D., Hoskin P.J., StalpersL.J.,Schulte R., Poortmans P., Veninga R., Schilds S.E., 92006). Short course radiotherapy is not optimal for spinal cord compression due to myeloma. International-1 Journal of Radiation Oncology Biology Physics, 64; 1452-1457.

[8] Mohty B., El-Cheikn J.,Jakoub-Agha J., Moreau P., Harounssean S., (2010). Peripheral neuropathy and new treatments in multiple myeloma: background and practical recommendations. Haematologica, 95, 311319.

[9] Smith A., Wisloff F. and Samson B., (2006). Guidelines on diagnosis and management of multiple myeloma 2005. British Journal of Hematology 132, 410-451.

[10] Terpos E., Dimopolous M.A. (2005). Myeloma bone disease: pathophysiology and management. Annals of Oncology 16, 1223-1231.

[11]Elektherianis-Papiakovou V., Bamias A., Gikas D., Simeonidis A., Pouli A., Anagnostopolous A., Michali E., Economopulus T., Dimopulos MA.,(2007). Renal failure in myeloma multiplex: incidence, correlations and prognostic significance. Leukemia and Lymphoma, 48 337-341.

[12]Facon, T., Mary, J.Y., Hulin, C., Benboubker, L., Attal, M., Pegourie, B., Renaud, M., Harousseau, J.L., Guillerm, G., Chaleteix, C., Dib, M., Voillat, L., Maisonneuve, H., Troncy, J., Dorvaux, V., Monconduit, M., Martin, C., Casassus, P., Jaubert, J., Jardel, H., Doyen, C., Kolb, B., Anglaret, B., Grosbois, B., Yakoub-Agha, I., Mathiot, C. \& Avet-Loiseau, H. (2007) Melphalan and prednisone plus thalidomide versus melphalan and prednisone alone or reducedintensity autologous stem cell transplantation in elderly patients with multiple myeloma (IFM 99-06): a randomised trial. Lancet, 370, 1209-1218.

[13] Myeloma Trialists' Collaborative Group. (1998) Combination chemotherapy versus melphalan plus prednisone as treatment for multiple myeloma: an overview of 6,633 patients from 27 randomized trials. Journal of Clinical Oncology, 16, 3832-3842.

[14] Rajkumar, S.V., Blood, E., Vesole, D., Fonseca, R. \& Greipp, P.R. (2006) Phase III clinical trial of thalidomide plus dexamethasone compared with dexamethasone alone in newly diagnosed multiple myeloma: a clinical trial coordinated by the Eastern
Cooperative Oncology Group. Journal of Clinical Oncology, 24, 431-436.

[15] Ludwig H., Drach J.,Tothova E., Grislinger H., Jaksic B., Linkesch W., Hajek R., Greil R.,Fridrik M., Labar B., Zojek N., Thalidomide-Dexamethasone versus Melphalan-Prednisolon as first line treatment in elderly patients with multiple myeloma: An interim analysis, Journal of Clinical Oncology, 2005 ASCO annual MeetingProseedings Vol.23, N16S (June 1 Suppl.)2005:6537.

[16] Palumbo, A., Bringhen, S., Liberati, A.M., Caravita, T., Falcone, A., V., C., Montanaro, M., Ria, R., Capaldi, A., Zambello, R., Benevolo, G., Derudas, D., Dore, F., Cavallo, F., Gay, F., Falco, P., Ciccone, G., Musto, P., Cavo, M. \& Boccadoro, M. (2008a) Oral melphalan, prednisone, and thalidomide in elderly patients with multiple myeloma: updated results of a randomized controlled trial. Blood, 112, 3107-3114.

[17] Kumar, S., Dispenzieri, A., Lacy, M.Q., Hayman, S.R., Buadi, F.K., Gastineau, D.A., Litzow, M.R., Fonseca, R., Roy, V., Rajkumar, S.V. \& Gertz, M.A. (2007) Impact of lenalidomide therapy on stem cell mobilization and engraftment post-peripheral blood stem cell transplantation in patients with newly diagnosed myeloma. Leukemia, 21, 2035-2042

[18] Alexanian, R., Barlogie, B. \& Tucker, S. (1990) VADbased regimens as primary treatment for multiple myeloma. American Journal of Hematology, 33, 86-89.

[19] Cavo, M., Zamagni, E., Tosi, P., Tacchetti, P., Cellini, C., Cangini, D., de Vivo, A., Testoni, N., Nicci, C., Terragna, C., Grafone, T., Perrone, G., Ceccolini, M., Tura, S. \& Baccarani, M. (2005) Superiority of thalidomide and dexamethasone over vincristinedoxorubicindexamethasone (VAD) as primary therapy in preparation for autologous transplantation for multiple myeloma. Blood, 106, 35-39.

[20] Barlogie, B., Tricot, G., Anaissie, E., Shaughnessy, J., Rasmussen, E., van Rhee, F., Fassas, A., Zangari, M., Hollmig, K., Pineda-Roman, M., Lee, C., Talamo, G., Thertulien, R., Kiwan, E., Krishna, S., Fox, M. \& Crowley, J. (2006b) Thalidomide and hematopoieticcell transplantation for multiple myeloma. New England Journal of Medicine, 354, 1021-1030.

[21]De Weers M., Tai YT, Vander Veer MS. Et al. Daratumumab, a novel therapeutic human CD38 monoclonal antibody, induces killing of multiple myeloma and other hematological tumors. J Immunol. $2011 ; 186: 1840-1848$.

\section{Volume 4 Issue 11, November 2015}

